Editorial published in Journal of Quantitative Spectroscopy and Radiative Transfer Citation:

Gérard Gouesbet, Fabrice R.A. Onofri,

Special issue: Laser-light and interactions with particles (LIP), 2020, Journal of Quantitative Spectroscopy and Radiative Transfer,

Volume 274, 2021, 107857, ISSN 0022-4073, https://doi.org/10.1016/j.jqsrt.2021.107857

(https://www.sciencedirect.com/science/article/pii/S0022407321003502)

\title{
Editorial
}

\section{Special issue: Laser-light and interactions with particles (LIP), 2020}

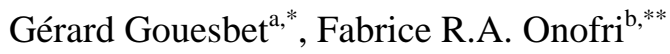 \\ du Madrillet, Saint-Etienne-du-Rouvray, 76800, France \\ bAix-Marseille Université, CNRS, IUSTI UMR 7343, 13 453, Marseille, France \\ *gouesbet@coria.fr \\ **fabrice.onofri@univ-amu.fr
}

aCORIA-UMR 6614, Normandie Université, CNRS, Université et INSA de Rouen, Campus Universitaire

This special issue contains a collection of research papers initially intended to be presented in 2020 at the international conference series on "Laser-light and Interactions with Particles (LIP)" which was postponed, due to the Covid pandemic.

Conference or not, the pandemic has not stopped Science. We think that this special issue will help to promote exchanges and trigger new ideas in the field of Laser-light and Interactions with Particles, on the modelling and experimental aspects, as well as its numerous applications in other research fields (e.g. atmospheric, soft matter and plasma physics, biology, combustion, multiphase flows) .

The 26 papers selected are introduced and commented in a preface to which the reader is kindly referred; see Volume 268, July 2021, 107635. They are essentially dealing with the electromagnetic and physical optics description of laser beams (Bessel-Gaussian, elliptical Bessel, Laguerre-Gaussian, Gaussiancorrelated), mechanical and thermal effects of light (Rayleigh scatter approximation, Optical tweezers, photophoresis), light scattering by particles and optical characterization methods (nano- to micro-particles, aggregates, propagation in turbulent atmospheres, photonic jet).

As guest editors and on behalf of the committees of LIP conferences, we want to deeply thank and honor the memory of Dr. Michael I. Mishchenko, Editor-in-Chief, who has initiated this special issue. Our thanks also go to the JQSRT editorial board, for the quality of their work and their unwavering support. We finally thank all authors of selected papers presented herein.

The 13th "Laser-light and Interactions with Particles, Optical Particle Characterization follow-up (LIP2022)" will be organized, in Warsaw, Poland, on August 21-26th, see at http://lip-conference.org/, with the help of the present authors and the conference Chairs Daniel Jakubczyk and Maciej Kolwas from the Institute of Physics, Polish Academy of Sciences. 


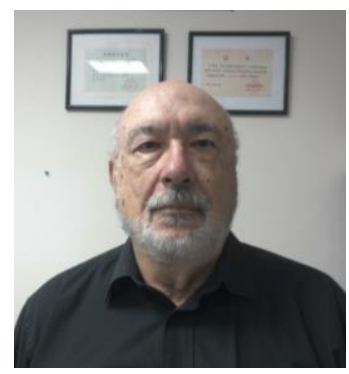

Gérard Gouesbet is a Professor Emeritus of the National Institute of Applied Sciences of Rouen. He has worked in particular on the modeling of turbulent and multiphase flows, on the nonlinear dynamics of systems, on combustion and plasmas, on optical particle characterization and light scattering. He is the driving force behind the development of generalized Lorenz-Mie theories, and the winner of the 2020 van de Hulst award. He is also involved in philosophical studies, e.g. his book in philosophy of sciences entitled "Hidden world in quantum physics, Dover publications" and his recently achieved tetralogy on the philosophy of violence (written in French)

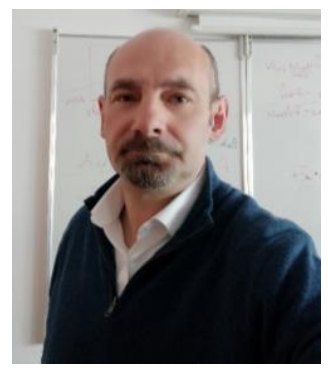

Fabrice Onofri is a research director at the French National Center for Scientific Research (CNRS). His research mainly focuses on the optical and electromagnetic characterization of particle and particle systems. Over the years, his research has found applications in various fields ranging from aerosols and multiphase flows, to soft matter and plasma physics, in the frame of national and international projects as well as industrial granted projects. He earned his Ph.D. degree at the University of Rouen and his habilitation degree at Aix-Marseille University, France. 